\title{
BENCHMARK RESULTS FOR PARTICLE TRANSPORT IN A BINARY MARKOV STATISTICAL MEDIUM
}

\author{
M. L. ADAMS \\ Lawrence Livermore National Laboratory, Livermore, CA 94550, U S.A \\ E. W. LARSEN \\ Department of Nuclear Engıneering, The University of Michigan, Ann Arbor, MI 48109, U.S.A. \\ G. C. Pomraning $\dagger$ \\ School of Engineering and Applied Science, Unıversity of Calıfornia at Los Angeles, \\ Los Angeles, CA 90024, U S A \\ (Recewed 27 January 1989)

\begin{abstract}
We give numerical benchmark results for partıcle transport in a randomly mixed binary medium, with the mixing statıstics described as a homogeneous Markov process. A Monte Carlo procedure is used to generate a physical realization of the statistics, and a discrete ordinate numerical transport solution is generated for this realization. The ensemble averaged solution, as well as the variance, is obtained by averaging a large number of such calculations. Reflection and transmission results are given for several problems in both rod and planar geometry. In a separate development, two coupled transport equations are derived which formally describe transport in a random binary mixture for arbitrary mixing statistics. Closing these equations by approximatıng their coupling terms in a low order and intuitive way leads to a model for stochastic transport previously obtaned via the master equation. The present derivation, based upon approximating exact equations, allows in principle the opportunity to develop more accurate models by making higher order approximations in the coupling terms.
\end{abstract}

\section{INTRODUCTION}

In the last 3 years, a fair number of papers have been written dealing the the problem of describing linear transport theory in a statistical medium consıstıng of two imiscible fluids. ${ }^{1-13}$ If we write the underlying transport equation as

$$
\begin{array}{r}
v^{-1} \partial \psi(\mathbf{r}, \mathbf{\Omega}, t) / \partial t+\mathbf{\Omega} \cdot \nabla \psi(\mathbf{r}, \boldsymbol{\Omega}, t)+\sigma(\mathbf{r}, t) \psi(\mathbf{r}, \mathbf{\Omega}, t) \\
=\left[\sigma_{\mathrm{s}}(\mathbf{r}, t) /(4 \pi)\right] \int_{4 \pi} \mathrm{d} \boldsymbol{\Omega}^{\prime} \psi\left(\mathbf{r}, \boldsymbol{\Omega}^{\prime}, t\right)+S(\mathbf{r}, \boldsymbol{\Omega}, t),
\end{array}
$$

the effort has been to develop a formalism to describe $\psi$, the ensemble-averaged solution to Eq. (1), when $\sigma, \sigma_{\mathrm{s}}$, and $S$ are two-state discrete random variables. Here $\psi(\mathbf{r}, \Omega, t)$ is the product of the particle distribution function and the particle speed $v$, with $\mathbf{r}, \boldsymbol{\Omega}$, and $t$ representing the spatial, angular, and temporal coordinates; $\sigma(\mathbf{r}, t)$ is the total (collision) cross section; $\sigma_{\mathrm{s}}(\mathbf{r}, t)$ is the scattering cross section; and $S(\mathbf{r}, \mathbf{\Omega}, t)$ represents any external source of particles. In writing Eq. (1) we have, for simplicity, restricted our attention to monoenergetic transport with isotropic scattering, but such restrictions are not essential to this work or the work reported in the literature. ${ }^{1-13}$ In treating stochastic transport associated with Eq. (1), the published works have assumed that the medium in which the transport occurs is composed of a random mixture of two materials, say 0 and 1 . Each material has a well defined nonstochastic source $S_{1}(\mathbf{r}, \mathbf{\Omega}, t)$ and cross sections $\sigma_{t}(\mathbf{r}, t)$ and $\sigma_{s t}(\mathbf{r}, t), i=0,1$. The stochasticity in the problem arises from the probabalistic nature of which material is present at any space-time point in the medium. The statistics of the mixture are assumed completely known.

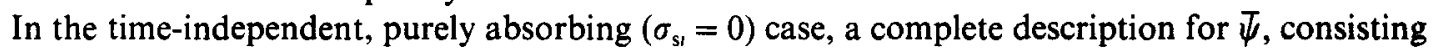
of two coupled first order ordinary differential equations, is known in the special case of Markov

†To whom all correspondence should be addressed at. 6266 Boelter Hall, UCLA-Engineerıng, Los Angeles, CA 90024, U S A 
mixıng statistics. ${ }^{1.2 .5}$ For non-Markov statıstics, the theory of alternating renewal processes has been used to again treat the time-independent, purely absorbing problem..$^{3-5,12,13}$ In this case one obtains an integral equation formulation, but for a certain restricted class of statistics describing the binary mixture these integral equations can be reduced to differential equations. ${ }^{5.13}$ If scattering is present in the underlying transport problem, the only exact formulation available corresponds to the case when $\sigma_{\mathrm{st}} / \sigma_{l}$ and $S_{l} / \sigma_{t}$ are nonstochastic; that is, these two ratios are both independent of the index 1 . Further, this formulation only applies to time-independent transport in one dimensional (rod and planar) geometries. ${ }^{7}$ Explicit results have only been obtained in the purely scattering case in finite rod geometry, ${ }^{6,7}$ and in half and full space geometries. ${ }^{8,9}$

A phenomenological model has been suggested to treat the general problem, including time dependence and scattering, in the case of Markov statistics. $4,5,10,11$ This model, first derived by applying the master equation approach, ${ }^{2-6}$ is known to be an approximate description, but it is a robust model which seems to be semıquantitatively accurate, based upon a single comparison with exact results in a purely scattering, time-independent, rod geometry transmission problem. ${ }^{7}$ This model states that $\psi$, the ensemble-averaged solution to Eq. (1), is given by

$$
\bar{\psi}=p_{0} \psi_{0}+p_{1} \psi_{1},
$$

where $\psi_{0}$ and $\psi_{1}$ satisfy the coupled set of transport equations

$$
\begin{aligned}
& v^{\prime} \partial\left(p, \psi_{i}\right) / \partial t+\boldsymbol{\Omega} \nabla\left(p_{t} \psi_{l}\right)+\sigma_{l} p_{t} \psi_{l} \\
& =\left[\sigma_{\mathrm{s} i} /(4 \pi)\right] \int_{4 \pi} \mathrm{d} \boldsymbol{\Omega}^{\prime} p_{i} \psi_{l}\left(\boldsymbol{\Omega}^{\prime}\right)+p_{l} S_{l}+p_{J} \psi_{J} / \lambda_{J}-p_{\imath} \psi_{\imath} / \lambda_{l}, \quad i, j=0,1, j \neq l .
\end{aligned}
$$

Here $\psi_{1}(\mathbf{r}, \boldsymbol{\Omega}, t)$ is the ensemble averaged intensity, given that position $\mathbf{r}$ is in material $i$ at time $t$; and $p_{t}(\mathbf{r}, t)$ is the probability that the space-time point $\mathbf{r}, t$ is in material $t$. The $\lambda_{t}$ in Eq. (3) are the Markov transition probabilities which, in general, depend upon $\mathbf{r}, \boldsymbol{\Omega}$, and $t$. They are defined as follows. If the medium is composed of material $i$ at some space-time point $s$, then the probability of the medium being in the other material a differential distance $\mathrm{d} s$ in direction $\Omega$ away from $s$ is given by $\mathrm{d} s / \lambda_{t}$. This Markov model has been extended to a certain class of non-Markov statistics, and the result is again two coupled equations of the same general form as given by Eq. (3). ${ }^{4}$

It seems clear that the complexity of stochastic transport theory will preclude an exact description for $\bar{\psi}$ in full generality including time dependence and scattering, even in the simplest case of Markov statistics, except perhaps in some abstract setting not suitable for computation. Thus from a practical point of view, a usable description will undoubtedly involve an approximate model. One such model is that represented by Eqs. (2) and (3). To test the accuracy of any such model, it is essential to have exact benchmark results. In this paper we make a first step in providing some meaningful benchmark results. We consider time-independent stochastic transport including scattering, under the assumption of Markov mixing statistics for the two components of the random medium. Further, the statistics are taken as homogeneous, by which we mean that all points in the system have the same statistical properties. We consider rod geometry, in which particles are constrained to move along a line, as well as layered planar geometry. We populate the medium statistically via a Monte Carlo procedure to affect a given physical realization of the statistics. Given this realization, we numerically solve the transport equation. We repeat this procedure a large number of times and average the results to obtain $\psi$, the ensemble averaged intensity. As part of this computational process, we also compute the variance of the solution, which gives an indication of the spread of the stochastic solution about the mean. The physical problem we consider is the transmission-reflection problem for a finite, source-free system, and we report the probabilities of transmission and reflection for a variety of choices for $\sigma_{l}, \sigma_{\mathrm{st}}, \lambda_{l}$, and system thickness. We also compare the predictions of Eqs. (2) and (3) with these benchmark results.

A second contribution of this paper is to provide a very simple derivation of a set of two coupled exact equations describing in complete generality particle transport in a binary statistical mixture for arbitrary mixing statistics. All of the statistical complexity is contained in coupling terms which need to be evaluated (approximated) to close these equations and turn them into a useful computational model. It is shown that one simple approximation for treating these coupling terms leads to the previously suggested model given by Eqs. (2) and (3). In contrast to the master equation 
derivation of this model, the present derivation holds out the hope of being able to improve upon this model since it is obtained from a simple approximation to an exact set of equations. A better approximation should lead to a better model. The use of the master equation, including time dependence and scattering, is in itself an approximation, and hence there seems to be no avenue available to improve upon the master equation derivation. We hope to address in a future paper improved approximations based upon the exact equations we present here.

\section{BENCHMARK RESULTS-ROD GEOMETRY}

We consider time-independent transport in rod geometry with no external source $(S=0)$. By rod geometry we mean that the particles are constrained to move along a line, which we take to be the $z$ axis. We assume isotropic scattering, which in this rod model implies that a particle, upon scattering, has an equal probability, (namely $1 / 2$ ) of continuing its direction of travel, or reversing its direction. The transport description for this situation is given by the two coupled equations

$$
\begin{aligned}
\mathrm{d} \psi^{+}(z) / \mathrm{d} z+\sigma(z) \psi^{+}(z) & =\sigma_{\mathrm{s}}(z)\left[\psi^{+}(z)+\psi^{-}(z)\right] / 2, \\
-\mathrm{d} \psi^{-}(z) / \mathrm{d} z+\sigma(z) \psi^{-}(z) & =\sigma_{\mathrm{s}}(z)\left[\psi^{+}(z)+\psi^{-}(z)\right] / 2,
\end{aligned}
$$

where $\psi^{ \pm}(z)$ is the intensity moving in the $\pm z$ direction. We assume Eqs. (4) and (5) hold for a rod of length $s$, and we assign nonstochastic boundary conditions of the form

$$
\psi^{+}(0)=1, \quad \psi^{-}(s)=0 .
$$

These boundary conditions correspond to a unit intensity incident upon the rod at $z=0$, with no intensity incident upon the rod at $z=s$.

We take this rod to be statistically composed of alternating segments of two materials, labeled 0 and 1 . Each material has spatially independent cross sections denoted by $\sigma_{1}$ and $\sigma_{\mathrm{st}}, i=0,1$. The statistics of this situation is assumed to be a homogeneous Markov process. It is known ${ }^{1,5,13}$ that this implies that the length of each segment of material $i$ is chosen at random from an exponential distribution given by

$$
f_{1}(\xi)=\lambda_{t}^{-1} \exp \left(-\xi / \lambda_{1}\right)
$$

Here $f_{i}(\xi) \mathrm{d} \xi$ is the probability of a segment of material $i$ having a length lying between $\xi$ and $\xi+\mathrm{d} \xi$, with $\lambda$, denoting the mean (average) segment length, i.e.,

$$
\lambda_{i}=\int_{0}^{\infty} \mathrm{d} \xi \xi f_{i}(\xi)
$$

At any point in the rod, the probability $p_{\imath}$ of finding material $i$ is given by

$$
p_{i}=\lambda_{1} /\left(\lambda_{0}+\lambda_{1}\right)
$$

To obtain ensemble-averaged results for this transport problem, we proceed as follows. We first generate a given physical realization of the statistics using a Monte Carlo procedure. Specifically, we choose the material present at $z=0$ statistically according to the probabilities $p_{1}$. We then sample from Eq. (7) for the value of $i$ so determined to establish the length of the first segment of material $i$, with its left-hand boundary at $z=0$. We next sample from Eq. (7) with the other material index to determine the length of the next segment of material. We then sample from Eq. (7) with the original index $i$ to determine the length of the third segment. We continue this process of sampling from Eq. (7) with alternating material indices until the entire interval $0 \leqslant z \leqslant s$ is populated with alternating segments of the two materials. This process yields both $\sigma(z)$ and $\sigma_{\mathrm{s}}(z)$ as particular histograms for this physical realization of the statistics. For this realization of the functions $\sigma(z)$ and $\sigma_{\mathrm{s}}(z)$, we numerically solve the transport problem given by Eqs. (4)-(6).

We used the well known diamond spatial differencing scheme ${ }^{14}$ to obtain this solution, with each material segment subdivided into smaller mesh intervals for numerical accuracy reasons. The size of each mesh interval in a given material segment was taken as a constant, not exceeding one-fifth of a mean free path, and each material segment contained at least one mesh interval. Specifically, we computed the probability of reflection $R$ and transmission $T$ for the rod, as given by

$$
R=\psi^{-}(0) ; \quad T=\psi^{+}(s) .
$$


The probability of absorption, $A$, in the rod follows from particle conservation, i.e.,

$$
R+T+A=1 .
$$

This entire procedure was repeated a large number of times to obtain results for a large number of physical realizations of the statistics. The ensemble-averaged solutions for the rod reflection and transmission, which we denote by $\bar{R}$ and $\bar{T}$, were then computed as simple numerical averages, i.e.,

$$
\bar{R}=M^{-1} \sum_{m=1}^{M} R_{m}, \quad \bar{T}=M^{-1} \sum_{m=1}^{M} T_{m},
$$

where the subscript $m$ on $R_{m}$ and $T_{m}$ is an index denoting a particular realization of the statistics, and $M$ denotes the number of realizations computed We also calculated the standard deviation $\Sigma$ of these results according to

$$
\begin{aligned}
& \Sigma^{2}(R)=M^{-1} \sum_{m=1}^{M} R_{m}^{2}-\bar{R}^{2}, \\
& \Sigma^{2}(T)=M^{-1} \sum_{m=1}^{M} T_{m}^{2}-\bar{T}^{2},
\end{aligned}
$$

which gives an indication of the spread of the results, due to the statistical nature of the problem, about the means.

We have used this procedure to obtain benchmark results for all combinations of three different sets of $\sigma_{1}$ and $\lambda_{1}$, three different sets of the single scatter albedo $\sigma_{s i} / \sigma_{i}$, and three different rod lengths $s$ Thus in all we considered 27 different statistical transport problems. In each case, we chose $M=10^{5}$ as the number of physical realizations of the statistics used to compute the ensemble averages. These values for $\sigma_{1}$ and $\lambda_{1}$ are those used previously in other contexts, ${ }^{4,7}$ and in all cases they correspond to an ensemble-averaged cross section, $\bar{\sigma}$, equal to unity. The results are given in Tables 1-9 under the headings Exact 1 and Exact 2. These two columns of results correspond to the use of two different sequences of random numbers to obtain the physical realizations of the statistics. The discrepancy between these two columns of results gives an indication of the adequacy of using $10^{5}$ realizations to compute ensemble averages. We typically see agreement between these two columns to somewhere between 2 and 3 significant figures. We also believe that our numerical discretization error in obtaining a solution for a given realization is of the same order, or less, than this statistical error. Thus we conclude that these benchmark results are accurate to somewhat

\begin{tabular}{|c|c|c|c|c|}
\hline & $\sigma_{0}=$ & & $\sigma_{1}=100 / 1$ & \\
\hline & $\sigma_{\mathrm{s} 0} / \sigma$ & $=0.00$ & $\sigma_{\mathbf{s}} / \sigma_{1}=1$ & \\
\hline & $\lambda_{0}=$ & $/ 100$ & $\lambda_{1}=11 / 10$ & \\
\hline $\mathbf{s}$ & & EXACT 1 & EXACT 2 & MODEL \\
\hline 01 & $\overline{\mathbf{R}}$ & 00344 & 00340 & 0.0337 \\
\hline & $\Sigma(R)$ & 0.0843 & 0.0841 & \\
\hline & $\overline{\mathbf{T}}$ & 09566 & 09569 & 09572 \\
\hline & $\Sigma(\mathrm{T})$ & 0.0818 & 00815 & \\
\hline 10 & $\overline{\mathbf{R}}$ & 0.2130 & 02123 & 01948 \\
\hline & $\Sigma(R)$ & 0.2100 & 02101 & \\
\hline & $\overline{\mathbf{T}}$ & 0.7006 & 07012 & 07187 \\
\hline & $\Sigma(T)$ & 0.1982 & 01982 & \\
\hline 100 & $\overline{\mathrm{R}}$ & & 04886 & 0.4393 \\
\hline & $\Sigma(\mathrm{R})$ & & 01313 & \\
\hline & $\overline{\mathrm{T}}$ & & 00526 & 00772 \\
\hline & $\Sigma(\mathrm{T})$ & & 00339 & \\
\hline
\end{tabular}
better than $1 \%$. Better accuracy could be achieved, of course, by using a larger value of $M$ and

Table 1. Reflection and transmission results for rod geometry 
Table 2 Reflection and transmission results for rod geometry.

\begin{tabular}{|c|c|c|c|c|}
\hline & \multicolumn{2}{|c|}{$\begin{array}{l}\sigma_{0}=10 / 99 \\
\sigma_{\mathrm{s} 0} / \sigma_{0}=100 \\
\lambda_{0}=99 / 100\end{array}$} & \multicolumn{2}{|c|}{$\begin{array}{l}\sigma_{1}=100 / 11 \\
\sigma_{s 1} / \sigma_{1}=000 \\
\lambda_{1}=11 / 100\end{array}$} \\
\hline $\mathbf{s}$ & & EXACT 1 & EXACT 2 & MODEL \\
\hline \multirow[t]{4}{*}{01} & $\overline{\mathrm{R}}$ & 00044 & 00045 & 00044 \\
\hline & $\Sigma(R)$ & 00014 & 00014 & \\
\hline & $\overline{\mathbf{T}}$ & 09285 & 09301 & 09293 \\
\hline & $\Sigma(\mathrm{T})$ & 01618 & 01603 & \\
\hline \multirow[t]{4}{*}{10} & $\overline{\mathbf{R}}$ & 00325 & 00326 & 00292 \\
\hline & $\Sigma(R)$ & 00159 & 00159 & \\
\hline & $\overline{\mathrm{T}}$ & 05699 & 05708 & 05699 \\
\hline & $\Sigma(\mathbf{T})$ & 03494 & 03486 & \\
\hline \multirow[t]{4}{*}{100} & $\overline{\mathbf{R}}$ & & 00615 & 00443 \\
\hline & $\Sigma(R)$ & & 00486 & \\
\hline & $\overline{\mathbf{T}}$ & & 00047 & 00045 \\
\hline & $\Sigma(\mathbf{T})$ & & 00223 & \\
\hline
\end{tabular}

Table 3. Reflection and transmission results for rod geometry

\begin{tabular}{|c|c|c|c|c|}
\hline & \multicolumn{2}{|c|}{$\begin{array}{l}\sigma_{0}=10 / 99 \\
\sigma_{s 0} / \sigma_{0}=090 \\
\lambda_{0}=99 / 100\end{array}$} & \multicolumn{2}{|c|}{$\begin{array}{l}\sigma_{1}=100 / 11 \\
\sigma_{s 1} / \sigma_{1}=0.90 \\
\lambda_{1}=11 / 100\end{array}$} \\
\hline $\mathbf{s}$ & & EXACT 1 & EXACT 2 & MODEL \\
\hline \multirow[t]{4}{*}{01} & $\overline{\mathbf{R}}$ & 00339 & 00332 & 00333 \\
\hline & $\Sigma(\mathbf{R})$ & 00717 & 00710 & \\
\hline & $\overline{\mathrm{T}}$ & 09563 & 09572 & 0.9570 \\
\hline & $\Sigma(\mathrm{T})$ & 00938 & 00930 & \\
\hline \multirow[t]{4}{*}{10} & $\overline{\mathbf{R}}$ & 02120 & 02121 & 01929 \\
\hline & $\Sigma(\mathbf{R})$ & 01586 & 01584 & \\
\hline & $\overline{\mathrm{T}}$ & 07017 & 07017 & 07182 \\
\hline & $\Sigma(T)$ & 02437 & 02432 & \\
\hline \multirow[t]{4}{*}{100} & $\overline{\mathbf{R}}$ & & 05146 & 04338 \\
\hline & $\Sigma(\mathbf{R})$ & & 00123 & \\
\hline & $\overline{\mathrm{T}}$ & & 00557 & 00759 \\
\hline & $\Sigma(T)$ & & 00623 & \\
\hline
\end{tabular}

more mesh intervals in the numerical solution. We also comment that we have used this numerical procedure to compute known results in the literature, namely the purely scattering rod problem ${ }^{6,7}$ and the purely absorbing rod problem for which analytic results are available. ${ }^{1,2}$ Comparison with these results led to the same conclusion regarding the accuracy of these benchmark results given in Tables 1-9; we found agreement to somewhere between 2 and 3 significant figures.

Also included in Tables 1-9, in the last column labeled Model, are the predictions of the model given by Eqs. (2) and (3). Specifically, for this rod problem the model transport equations are written

$$
\begin{aligned}
\mathrm{d} \psi_{l}^{+}(z) / \mathrm{d} z+\sigma_{l} \psi_{l}^{+}(z) & =\sigma_{\mathrm{sl}}\left[\psi_{1}^{+}(z)+\psi_{i}^{-}(z)\right] / 2+\lambda_{1}^{-1}\left[\psi_{J}^{+}(z)-\psi_{i}^{+}(z)\right], \\
-\mathrm{d} \psi_{i}^{-}(z) / \mathrm{d} z+\sigma_{\imath} \psi_{1}^{-}(z) & =\sigma_{\mathrm{sl}}\left[\psi_{1}^{+}(z)+\psi_{i}^{-}(z)\right] / 2+\lambda_{l}^{-1}\left[\psi_{J}^{-}(z)-\psi_{i}^{-}(z)\right],
\end{aligned}
$$

Table 4 Reflection and transmission results

\begin{tabular}{|c|c|c|c|c|}
\hline & \multicolumn{2}{|c|}{$\begin{array}{l}\sigma_{0}=10 / 99 \\
\sigma_{\mathrm{so}} / \sigma_{0}=0.00 \\
\lambda_{0}=99 / 10\end{array}$} & \multicolumn{2}{|c|}{$\begin{array}{l}\sigma_{1}=100 / 11 \\
\sigma_{31} / \sigma_{1}=100 \\
\lambda_{1}=11 / 10\end{array}$} \\
\hline $\mathrm{s}$ & & EXACT 1 & EXACT 2 & MODEL \\
\hline \multirow[t]{4}{*}{01} & $\overline{\mathbf{R}}$ & 00319 & 00316 & 0.0315 \\
\hline & $\boldsymbol{\Sigma}(\mathbf{R})$ & 0.0931 & 00927 & \\
\hline & $\overline{\mathbf{T}}$ & 0.9591 & 09594 & 09594 \\
\hline & $\Sigma(T)$ & 0.0901 & 00897 & \\
\hline \multirow[t]{4}{*}{10} & $\overline{\mathbf{R}}$ & 01130 & 01105 & 01003 \\
\hline & $\boldsymbol{\Sigma}(\mathbf{R})$ & 02531 & 0.2511 & \\
\hline & $\overline{\mathbf{T}}$ & 08006 & 0.8029 & 0.8132 \\
\hline & $\Sigma(T)$ & 0.2295 & 0.2275 & \\
\hline \multirow[t]{4}{*}{100} & $\overline{\mathbf{R}}$ & & 0.2841 & 0.2158 \\
\hline & $\Sigma(R)$ & & 02876 & \\
\hline & $\tilde{\mathbf{T}}$ & & 0.1774 & 0.2294 \\
\hline & $\Sigma(T)$ & & 0.1437 & \\
\hline
\end{tabular}
for rod geometry
Table 5 Reflection and transmissıon results

\begin{tabular}{|c|c|c|c|c|}
\hline & \multicolumn{2}{|c|}{$\begin{array}{l}\sigma_{0}=10 / 99 \\
\sigma_{s 0} / \sigma_{0}=1.00 \\
\lambda_{0}=99 / 10\end{array}$} & \multicolumn{2}{|c|}{$\begin{array}{l}\sigma_{1}=100 / 11 \\
\sigma_{31} / \sigma_{1}=000\end{array}$} \\
\hline $\mathbf{s}$ & & EXACT 1 & EXACT 2 & MODEL \\
\hline \multirow[t]{4}{*}{01} & $\overline{\mathbf{R}}$ & 00045 & 0.0045 & 00045 \\
\hline & $\Sigma(R)$ & 0.0015 & 00015 & \\
\hline & $\overline{\mathrm{T}}$ & 09343 & 0.9349 & 09350 \\
\hline & $\Sigma(\mathbf{T})$ & 0.1768 & 0.1759 & \\
\hline \multirow[t]{4}{*}{10} & $\overline{\mathbf{R}}$ & 0.0414 & 0.0414 & 00402 \\
\hline & $\Sigma(\mathrm{R})$ & 0.0154 & 0.0154 & \\
\hline & $\overline{\mathbf{T}}$ & 0.7948 & 0.7961 & 0.7970 \\
\hline & $\Sigma(T)$ & 0.3384 & 0.3379 & \\
\hline \multirow[t]{4}{*}{100} & $\overline{\mathbf{R}}$ & & 0.2078 & 0.1568 \\
\hline & $\Sigma(R)$ & & 0.1243 & \\
\hline & $\overline{\mathbf{T}}$ & & 02430 & 02352 \\
\hline & $\Sigma(T)$ & & 0.3067 & \\
\hline
\end{tabular}
for rod geometry 
Table 6. Reflection and transmission results for rod geometry

\begin{tabular}{|c|c|c|c|c|}
\hline & \multicolumn{2}{|c|}{$\begin{array}{l}\sigma_{0}=10 / 99 \\
\sigma_{\mathrm{s} 0} / \sigma_{0}=090 \\
\lambda_{0}=99 / 10\end{array}$} & \multicolumn{2}{|c|}{$\begin{array}{l}\sigma_{1}=100 / 11 \\
\sigma_{\mathrm{s} 1} / \sigma_{1}=090 \\
\lambda_{1}=11 / 10\end{array}$} \\
\hline $\mathbf{s}$ & & EXACT 1 & EXACT 2 & MODEL \\
\hline \multirow[t]{4}{*}{01} & $\overline{\mathrm{R}}$ & 00315 & 00310 & 00311 \\
\hline & $\Sigma(R)$ & 00785 & 00779 & \\
\hline & $\overline{\mathrm{T}}$ & 09589 & 09595 & 09593 \\
\hline & $\Sigma(\mathrm{T})$ & 01039 & 01031 & \\
\hline \multirow[t]{4}{*}{10} & $\overline{\mathrm{R}}$ & 01181 & 01173 & 01045 \\
\hline & $\Sigma(\mathrm{R})$ & 01632 & 01627 & \\
\hline & $\overline{\mathrm{T}}$ & 08181 & 0.8194 & 08270 \\
\hline & $\Sigma(\mathrm{T})$ & 02873 & 02866 & \\
\hline \multirow[t]{4}{*}{100} & $\overline{\mathrm{R}}$ & & 04301 & 02883 \\
\hline & $\Sigma(\mathrm{R})$ & & 01066 & \\
\hline & $\overline{\mathrm{T}}$ & & 02658 & 02961 \\
\hline & $\Sigma(\mathbf{T})$ & & 02728 & \\
\hline
\end{tabular}

Table 7 Reflection and transmission results for rod geometry

\begin{tabular}{|c|c|c|c|c|}
\hline & $\sigma_{0}=2$ & & $\sigma_{1}=200 / 1$ & \\
\hline & $\sigma_{\mathrm{s} d} / \sigma_{0}$ & $=000$ & $\sigma_{\mathrm{sl}} / \sigma_{1}=1$ & \\
\hline & $\lambda_{0}=1$ & $1 / 20$ & $\lambda_{1}=101 / 2$ & \\
\hline$s$ & & EXACT 1 & EXACT 2 & MODEL \\
\hline 01 & $\overline{\mathbf{R}}$ & 00450 & 00454 & 00451 \\
\hline & $\Sigma(\mathrm{R})$ & 00448 & 00447 & \\
\hline & $\overline{\mathrm{T}}$ & 09540 & 09536 & 09539 \\
\hline & $\Sigma(\mathrm{T})$ & 00438 & 00438 & \\
\hline 10 & $\overline{\mathbf{R}}$ & 02586 & 02578 & 02562 \\
\hline & $\Sigma(\mathrm{R})$ & 02338 & 02340 & \\
\hline & $\overline{\mathrm{T}}$ & 07316 & 07324 & 07340 \\
\hline & $\Sigma(\mathrm{T})$ & 02247 & 02248 & \\
\hline 100 & $\overline{\mathbf{R}}$ & & 06804 & 06034 \\
\hline & $\Sigma(\mathrm{R})$ & & 02528 & \\
\hline & $\overline{\mathbf{T}}$ & & 02310 & 03063 \\
\hline & $\Sigma(\mathrm{T})$ & & 02029 & \\
\hline
\end{tabular}

for $i=0,1$ with $j \neq i$. The boundary conditions for Eqs. (15) and (16) are

$$
\psi_{\imath}^{+}(0)=1 ; \quad \psi_{\imath}^{-}(s)=0, \quad \imath=0,1,
$$

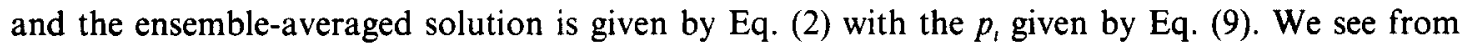
the numerical results that this model is quite accurate for small rods $(s=0.1)$. This is not unexpected since it can be shown that both the exact treatment and the model approach the atomic mix limit as $s$, the rod length, approaches zero. This limit corresponds to using the usual transport equation written for $\bar{\psi}$, with $\sigma, \sigma_{\mathrm{s}}$, and $S$ taken as ensemble averaged quantities. ${ }^{1.5}$ As the rod length $s$ increases, the accuracy of the model generally deteriorates, but it appears that the model is qualitatively as well as semiquantitatively correct. The overall results seem to indicate that the model generally underestimates the reflection and overestimates the transmission. The model

Table 8 Reflection and transmission results for rod geometry

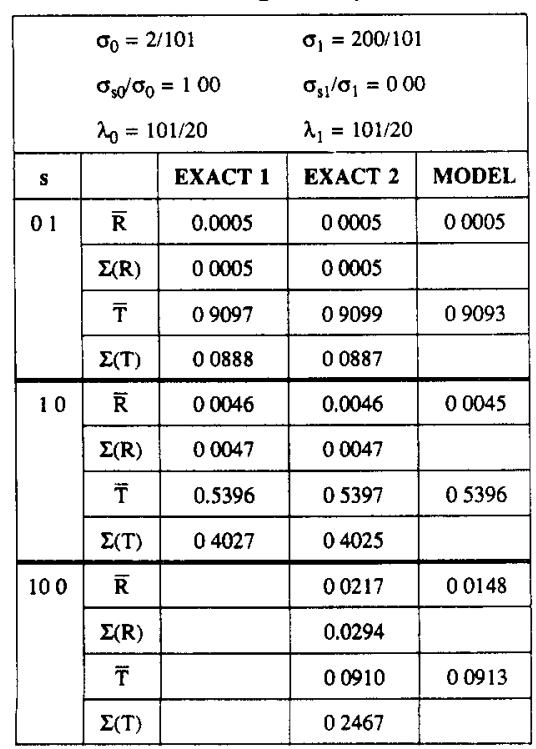

Table 9. Reflection and transmission results for rod geometry.

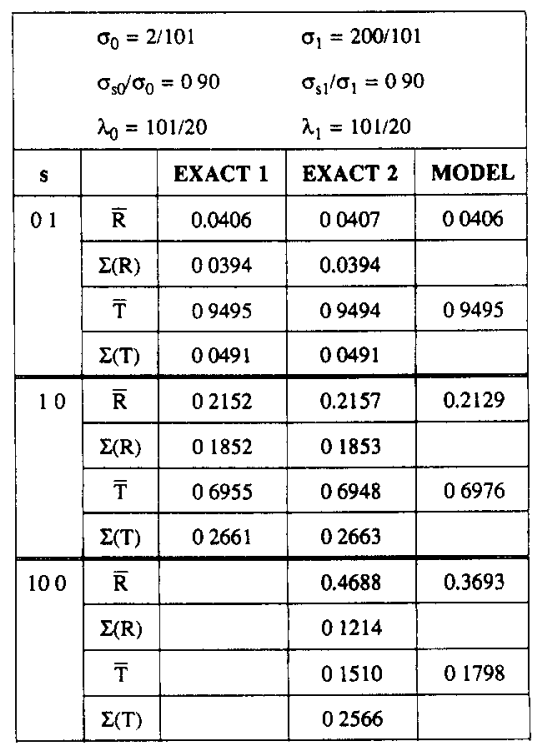


equations do not predict results for the standard deviations $\Sigma(R)$ and $\Sigma(T)$, although the master equation approach to deriving Eqs. (2) and (3) can be used to derive a model for these quantities. We do not pursue this here.

\section{BENCHMARK RESULTS-PLANAR GEOMETRY}

We now consider time-independent transport in planar geometry with no external source of particles $(S=0)$. If we assume isotropic scattering, the transport equation is written

$$
\mu \partial \psi(z, \mu) / \partial z+\sigma(z) \psi(z, \mu)=\left[\sigma_{\mathrm{s}}(z) / 2\right] \int_{-1}^{1} \mathrm{~d} \mu^{\prime} \psi\left(z, \mu^{\prime}\right),
$$

where $\mu$ here is the cosine of the angle between the $z$ axis and the particle flight direction. We take Eq. (18) to hold on the interval $0 \leqslant z \leqslant s$, and we assign nonstochastic boundary conditions given by

$$
\psi(0, \mu)=2, \quad \mu>0 ; \quad \psi(s, \mu)=0, \quad \mu<0 .
$$

These boundary conditions correspond to an isotropic intensity, normalized to a unit incoming flux, incident upon the planar system at $z=0$, and no intensity incident upon the system surface at $z=s$. We take this planar system to be statistically composed of alternating slabs of two materials, which we again label with indices 0 and 1 . As in the rod problem, each material has spatially independent cross sections $\sigma_{t}$ and $\sigma_{\mathrm{st}}$. The thickness of each slab is chosen at random from the exponential distribution given by Eq. (7). This statistical description corresponds to a homogeneous Markov process.

To obtain ensemble-averaged results for this problem, we proceed in complete analogy to the procedure used in rod geometry. We obtain a physical realization of the statistics via the Monte Carlo procedure of sampling the slab thicknesses from Eq. (7), and for this realization we solve the corresponding transport problem numerically. The angular variable $\mu$ in Eq. (18) was treated using the standard discrete ordinate method, ${ }^{14}$ employing the 16-point Gauss-Legendre quadrature set. The spatial differencing used was simple diamond differencing. ${ }^{14}$ The size $\Delta z$ of each mesh interval was chosen such that

$$
\sigma_{1} \Delta z /|\mu|_{\min } \leqslant 1 / 5
$$

where $|\mu|_{\min }$ is the smallest positive ordinate in the $S-16$ quadrature set. Just as in the rod problem, we computed the probabilities of reflection $R$ and transmission $T$ for the system, which in this case are given by

$$
R=\int_{0}^{1} \mathrm{~d} \mu \mu \psi(0,-\mu) ; \quad T=\int_{0}^{1} \mathrm{~d} \mu \mu \psi(s, \mu),
$$

and the absorption probability $A$ follows from particle conservation according to Eq. (11) Repeating this process for a large number of statistical realizations, ensemble-averaged results for the reflection and transmission follow from Eq. (12), and Eqs. (13) and (14) give the corresponding standard deviations. As in our rod calculations, we used $10^{5}$ realizations to compute the ensemble averages. These calculations, performed on a Cray XMP at the Lawrence Livermore National Laboratory, required approx. $10 \mathrm{~h}$ of cpu time.

Benchmark results for the same parameters $\sigma_{l}, \sigma_{s l}$, and $\lambda_{1}$ as we used in rod geometry are displayed in Tables 10-18. Aside from the angular discretization error inherent in the $S$-16 approximation, we believe these results to be accurate to somewhat better than $1 \%$, just as in rod geometry. The error associated with using the $S-16$ discrete ordinate method is probably in general of this same order, but may be larger for highly absorbing thick systems, in particular for the transmission $T$. Also included in these tables are the predictions of the model given by Eqs. (2) and (3). For the time-independent, source-free planar problem under consideration, Eq. (3) is written

$$
\mu \partial \psi_{i}(z, \mu) / \partial z+\sigma_{i} \psi_{l}(z, \mu)=\left[\sigma_{\mathrm{s} I} / 2\right] \int_{-1}^{1} \mathrm{~d} \mu^{\prime} \psi_{i}\left(z, \mu^{\prime}\right)+|\mu| \lambda_{l}^{-1}\left[\psi_{J}(z, \mu)-\psi_{i}(z, \mu)\right],
$$


Table 10 Reflection and transmission results for planar geometry.

\begin{tabular}{|l|c|l|l|}
\hline \multicolumn{2}{|l|}{$\sigma_{0}=10 / 99$} & \multicolumn{2}{l|}{$\sigma_{1}=100 / 11$} \\
$\sigma_{s 0} / \sigma_{0}=000$ & \multicolumn{2}{l|}{$\sigma_{\mathrm{s}} / \sigma_{1}=100$} \\
$\lambda_{0}=99 / 100$ & \multicolumn{2}{l|}{$\lambda_{1}=11 / 100$} \\
\hline$s$ & & EXACT & MODEL \\
\hline 01 & $\overline{\mathrm{R}}$ & 00491 & 00479 \\
\cline { 2 - 4 } & $\Sigma(\mathrm{R})$ & 01182 & \\
\hline & $\overline{\mathrm{T}}$ & 09331 & 09343 \\
\hline & $\Sigma(\mathrm{T})$ & 01132 & \\
\hline 10 & $\overline{\mathrm{R}}$ & 02495 & 0.2187 \\
\hline & $\Sigma(\mathrm{R})$ & 02354 & \\
\cline { 2 - 4 } & $\overline{\mathrm{T}}$ & 05950 & 06254 \\
\hline & $\Sigma(\mathrm{T})$ & 02143 & \\
\hline \multirow{2}{*}{100} & $\overline{\mathrm{R}}$ & 0.4342 & 03760 \\
\cline { 2 - 4 } & $\Sigma(\mathrm{R})$ & 01616 & \\
\hline & $\overline{\mathrm{T}}$ & 00146 & 00259 \\
\hline & $\Sigma(\mathrm{T})$ & 00152 & \\
\hline
\end{tabular}

Table 11 Reflection and transmission results for planar geometry

\begin{tabular}{|c|c|c|c|}
\hline \multicolumn{2}{|c|}{$\begin{array}{l}\sigma_{0}=10 / 99 \\
\sigma_{s 0} / \sigma_{0}=100 \\
\lambda_{0}=99 / 100\end{array}$} & \multicolumn{2}{|c|}{$\begin{array}{l}\sigma_{1}=100 / 11 \\
\sigma_{s 1} / \sigma_{1}=000 \\
\lambda_{1}=11 / 100\end{array}$} \\
\hline $\mathbf{s}$ & & EXACT & MODEL \\
\hline \multirow[t]{4}{*}{01} & $\overline{\mathbf{R}}$ & 00087 & 00086 \\
\hline & $\Sigma(\mathrm{R})$ & 00029 & \\
\hline & $\overline{\mathrm{T}}$ & 09014 & 09004 \\
\hline & $\Sigma(T)$ & 02111 & \\
\hline \multirow[t]{4}{*}{10} & $\overline{\mathbf{R}}$ & 00548 & 00460 \\
\hline & $\Sigma(\mathbf{R})$ & 00307 & \\
\hline & $\overline{\mathrm{T}}$ & 04841 & 04834 \\
\hline & $\Sigma(T)$ & 03632 & \\
\hline \multirow[t]{4}{*}{100} & $\overline{\mathrm{R}}$ & 00856 & 00591 \\
\hline & $\Sigma(\mathrm{R})$ & 00697 & \\
\hline & $\overline{\mathrm{T}}$ & 00016 & 00015 \\
\hline & $\Sigma(\mathrm{T})$ & 00121 & \\
\hline
\end{tabular}

Table 12 Reflection and transmission results for planar geometry

\begin{tabular}{|c|c|c|c|}
\hline \multicolumn{2}{|c|}{$\begin{array}{l}\sigma_{0}=10 / 99 \\
\sigma_{\mathrm{s} 0} / \sigma_{0}=090 \\
\lambda_{0}=99 / 100\end{array}$} & \multicolumn{2}{|c|}{$\begin{array}{l}\sigma_{1}=100 / 11 \\
\sigma_{s 1} / \sigma_{1}=090 \\
\lambda_{1}=11 / 100\end{array}$} \\
\hline $\mathbf{s}$ & & EXACT & MODEL \\
\hline \multirow[t]{4}{*}{01} & $\overline{\mathrm{R}}$ & 00480 & 00473 \\
\hline & $\Sigma(\mathrm{R})$ & 00935 & \\
\hline & $\overline{\mathrm{T}}$ & 09341 & 09344 \\
\hline & $\Sigma(\mathrm{T})$ & 01339 & \\
\hline \multirow[t]{4}{*}{10} & $\overline{\mathrm{R}}$ & 02563 & 02178 \\
\hline & $\Sigma(\mathrm{R})$ & 01546 & \\
\hline & $\overline{\mathbf{T}}$ & 05985 & 06267 \\
\hline & $\Sigma(\mathrm{T})$ & 0.2832 & \\
\hline \multirow[t]{4}{*}{100} & $\overline{\mathbf{R}}$ & 04785 & 03707 \\
\hline & $\Sigma(\mathrm{R})$ & 0.0038 & \\
\hline & $\overline{\mathbf{T}}$ & 00159 & 00237 \\
\hline & $\Sigma(\mathbf{T})$ & 00314 & \\
\hline
\end{tabular}

for $\iota=0,1$ with $j \neq l$. The boundary conditions on Eq. (22) are

$$
\psi_{1}(0, \mu)=2, \quad \mu>0 ; \quad \psi_{i}(s, \mu)=0, \quad \mu<0,
$$

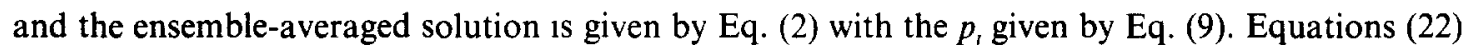
and (23) were solved numerically using the $S-16$ discrete ordinate approximation and diamond spatial differencing. ${ }^{14}$ The coupling term $|\mu| \lambda_{1}^{-1}$ in Eq. (22) deserves a word of explanation. If the mean (average) slab thickness of material $i$ is $\lambda_{i}$, then the mean chord length seen by a particle traveling at an angle characterized by its cosine $\mu$ is just $\lambda_{1} /|\mu|$. That is, the Markov transition probabilities are, in this case of a layered planar system, angularly dependent and given by $\lambda_{l} /|\mu|$, which leads to this form of the coupling term in Eq (22).

Table 13. Reflection and transmission results for planar geometry

\begin{tabular}{|c|c|c|c|}
\hline \multicolumn{2}{|c|}{$\begin{array}{l}\sigma_{0}=10 / 99 \\
\sigma_{30} / \sigma_{0}=0,00 \\
\lambda_{0}=99 / 100\end{array}$} & \multicolumn{2}{|c|}{$\begin{array}{l}\sigma_{1}=100 / 11 \\
\sigma_{s 1} / \sigma_{1}=100 \\
\lambda_{1}=11 / 100\end{array}$} \\
\hline s & & EXACT & MODEL \\
\hline \multirow[t]{4}{*}{0.1} & $\overline{\mathbf{R}}$ & 00434 & 00432 \\
\hline & $\Sigma(R)$ & 01267 & \\
\hline & $\overline{\mathrm{T}}$ & 09388 & 09390 \\
\hline & $\Sigma(T)$ & 01209 & \\
\hline \multirow[t]{4}{*}{1.0} & $\overline{\mathbf{R}}$ & 01224 & 0.1068 \\
\hline & $\Sigma(R)$ & 0.2726 & \\
\hline & $\overline{\mathbf{T}}$ & 07233 & 0.7385 \\
\hline & $\boldsymbol{\Sigma}(\mathrm{T})$ & 0.2306 & \\
\hline \multirow[t]{4}{*}{100} & $\overline{\mathbf{R}}$ & 02369 & 01799 \\
\hline & $\Sigma(R)$ & 02860 & \\
\hline & $\overline{\mathrm{T}}$ & 0.0981 & 01278 \\
\hline & $\Sigma(T)$ & 0.0887 & \\
\hline
\end{tabular}

Table 14 Reflection and transmission results for planar geometry

\begin{tabular}{|c|c|c|c|}
\hline \multicolumn{2}{|c|}{$\begin{array}{l}\sigma_{0}=10 / 99 \\
\sigma_{50} / \sigma_{0}=1.00 \\
\lambda_{0}=99 / 100\end{array}$} & \multicolumn{2}{|c|}{$\begin{array}{l}\sigma_{1}=100 / 11 \\
\sigma_{\mathrm{s} 1} / \sigma_{1}=000 \\
\lambda_{1}=11 / 100\end{array}$} \\
\hline $\mathrm{s}$ & & EXACT & MODEL \\
\hline \multirow[t]{4}{*}{01} & $\overline{\mathrm{R}}$ & 0.0089 & 00089 \\
\hline & $\Sigma(\mathbf{R})$ & 00030 & \\
\hline & $\overline{\mathrm{T}}$ & 09140 & 09140 \\
\hline & $\Sigma(\mathrm{T})$ & 0.2217 & \\
\hline \multirow[t]{4}{*}{10} & $\overline{\mathbf{R}}$ & 0.0744 & 00717 \\
\hline & $\Sigma(\mathbf{R})$ & 0.0278 & \\
\hline & $\overline{\mathrm{T}}$ & 0.7588 & 07581 \\
\hline & $\Sigma(T)$ & 0.3325 & \\
\hline \multirow[t]{4}{*}{10.0} & $\overline{\mathbf{R}}$ & 02897 & 0.2193 \\
\hline & $\Sigma(R)$ & 01631 & \\
\hline & $\overline{\mathbf{T}}$ & 01960 & 01787 \\
\hline & $\Sigma(\mathrm{T})$ & 02551 & \\
\hline
\end{tabular}

Table 15 Reflection and transmission results for planar geometry

\begin{tabular}{|c|c|c|c|}
\hline \multicolumn{2}{|c|}{$\begin{array}{l}\sigma_{0}=10 / 99 \\
\sigma_{\mathrm{s} 0} / \sigma_{0}=090 \\
\lambda_{0}=99 / 100\end{array}$} & \multicolumn{2}{|c|}{$\begin{array}{l}\sigma_{1}=100 / 11 \\
\sigma_{s 1} / \sigma_{1}=090 \\
\lambda_{1}=11 / 100\end{array}$} \\
\hline $\mathbf{s}$ & & EXACT & MODEL \\
\hline \multirow[t]{4}{*}{01} & $\overline{\mathbf{R}}$ & 0.0426 & 00426 \\
\hline & $\Sigma(\mathbf{R})$ & 00985 & \\
\hline & $\overline{\mathbf{T}}$ & 09398 & 09397 \\
\hline & $\Sigma(\mathrm{T})$ & 01446 & \\
\hline \multirow[t]{4}{*}{1.0} & $\overline{\mathbf{R}}$ & 01440 & 01255 \\
\hline & $\Sigma(\mathbf{R})$ & 01452 & \\
\hline & $\overline{\mathrm{T}}$ & 07666 & 0.7733 \\
\hline & $\Sigma(T)$ & 0.3011 & \\
\hline \multirow[t]{4}{*}{100} & $\overline{\mathbf{R}}$ & 04344 & 02910 \\
\hline & $\Sigma(\mathrm{R})$ & 0.0572 & \\
\hline & $\overline{\mathbf{T}}$ & 0.1861 & 01945 \\
\hline & $\Sigma(\mathrm{T})$ & 02134 & \\
\hline
\end{tabular}


Table 16. Reflection and transmission results for planar geometry

\begin{tabular}{|c|c|c|c|}
\hline \multicolumn{2}{|c|}{$\begin{array}{l}\sigma_{0}=2 / 101 \\
\sigma_{\mathrm{s} 0} / \sigma_{0}=000 \\
\lambda_{0}=101 / 20\end{array}$} & \multicolumn{2}{|c|}{$\begin{array}{l}\sigma_{1}=200 / 101 \\
\sigma_{31} / \sigma_{1}=100 \\
\lambda_{1}=101 / 20\end{array}$} \\
\hline$s$ & & EXACT & MODEL \\
\hline \multirow[t]{4}{*}{01} & $\overline{\mathbf{R}}$ & 00763 & 0.0758 \\
\hline & $\Sigma(\mathbf{R})$ & 00751 & \\
\hline & $\overline{\mathbf{T}}$ & 09218 & 0.9223 \\
\hline & $\Sigma(\mathrm{T})$ & 00732 & \\
\hline \multirow[t]{4}{*}{10} & $\overline{\mathbf{R}}$ & 03210 & 03157 \\
\hline & $\Sigma(R)$ & 02865 & \\
\hline & $\overline{\mathrm{T}}$ & 06599 & 06652 \\
\hline & $\Sigma(T)$ & 02688 & \\
\hline \multirow[t]{4}{*}{100} & $\overline{\mathbf{R}}$ & 06916 & 0.6070 \\
\hline & $\Sigma(R)$ & 02615 & \\
\hline & $\overline{\mathrm{T}}$ & 01615 & 02391 \\
\hline & $\Sigma(\mathrm{T})$ & 01740 & \\
\hline
\end{tabular}

Table 17. Reflection and transmission results for planar geometry.

\begin{tabular}{|c|c|c|c|}
\hline \multicolumn{2}{|c|}{$\begin{array}{l}\sigma_{0}=2 / 101 \\
\sigma_{s} \sigma_{0}=1.00 \\
\lambda_{0}=101 / 20\end{array}$} & \multicolumn{2}{|c|}{$\begin{array}{l}\sigma_{1}=200 / 101 \\
\sigma_{s 1} / \sigma_{1}=000 \\
\lambda_{1}=101 / 20\end{array}$} \\
\hline $\mathbf{s}$ & & EXACT & MODEL \\
\hline \multirow[t]{4}{*}{01} & $\overline{\mathbf{R}}$ & 00010 & 00010 \\
\hline & $\Sigma(R)$ & 00010 & \\
\hline & $\overline{\mathbf{T}}$ & 08509 & 08503 \\
\hline & $\Sigma(\mathrm{T})$ & 01464 & \\
\hline \multirow[t]{4}{*}{10} & $\overline{\mathbf{R}}$ & 00088 & 0.0085 \\
\hline & $\Sigma(\mathrm{R})$ & 00091 & \\
\hline & $\overline{\mathbf{T}}$ & 04818 & 04826 \\
\hline & $\Sigma(\mathrm{T})$ & 04361 & \\
\hline \multirow[t]{4}{*}{100} & $\overline{\mathbf{R}}$ & 00369 & 00243 \\
\hline & $\Sigma(R)$ & 00500 & \\
\hline & $\overline{\mathbf{T}}$ & 00766 & 00755 \\
\hline & $\Sigma(\mathrm{T})$ & 02252 & \\
\hline
\end{tabular}

Table 18. Reflection and transmission results for planar geometry

\begin{tabular}{|c|c|c|c|}
\hline \multicolumn{2}{|c|}{$\begin{array}{l}\sigma_{0}=2 / 101 \\
\sigma_{\mathrm{s} 0} / \sigma_{0}=0.90 \\
\lambda_{0}=101 / 20\end{array}$} & \multicolumn{2}{|c|}{$\begin{array}{l}\sigma_{1}=200 / 101 \\
\sigma_{s 1} / \sigma_{1}=090 \\
\lambda_{1}=101 / 20\end{array}$} \\
\hline $\mathbf{s}$ & & EXACT & MODEL \\
\hline \multirow[t]{4}{*}{01} & $\overline{\mathbf{R}}$ & 00670 & 00669 \\
\hline & $\Sigma(\mathbf{R})$ & 0.0646 & \\
\hline & $\overline{\mathbf{T}}$ & 09136 & 09137 \\
\hline & $\Sigma(\mathrm{T})$ & 00834 & \\
\hline \multirow[t]{4}{*}{10} & $\overline{\mathbf{R}}$ & 02435 & 02381 \\
\hline & $\Sigma(R)$ & 01991 & \\
\hline & $\overline{\mathbf{T}}$ & 06045 & 06086 \\
\hline & $\Sigma(\mathrm{T})$ & 03346 & \\
\hline \multirow[t]{4}{*}{100} & $\overline{\mathbf{R}}$ & 04466 & 03272 \\
\hline & $\Sigma(\mathbf{R})$ & 00923 & \\
\hline & $\overline{\mathbf{T}}$ & 01037 & 01195 \\
\hline & $\Sigma(\mathrm{T})$ & 02290 & \\
\hline
\end{tabular}

The numerical results given in Tables 10-18 for planar geometry follow the same general trends as the rod geometry results given in Tables 1-9. The model equations in general underestimate the ensemble averaged reflection probability $\bar{R}$ and overestimate the ensemble-averaged transmission probability $\bar{T}$, with the accuracy becoming better as the thickness of the system decreases.

\section{STATISTICAL TRANSPORT EQUATIONS}

In this section we give a simple derivation of two coupled, formally exact, transport equations describing particle flow in a binary statistical mixture. The mixing statistics are arbitrary, and assumed known. Our considerations are completely general in that they include time dependence and scattering in full three-dimensional geometry. We start by considering a particular physical realization of the statistics, and for this realization we consider an arbitrary convex volume $V$, bounded by the surface $B$, within the medium under consideration. In general, both materials, which we label by indices 0 and 1 , will be present within the volume $V$, and we define $\Gamma$ as the union of all surfaces in the interior of $V$ which separate materials 0 and 1 . Each material is characterized by a nonstochastic source $S_{i}(\mathbf{r}, \Omega, t)$ and nonstochastic cross sections $\sigma_{t}(\mathbf{r}, t)$ and $\sigma_{\mathrm{sl}}(\mathbf{r}, t)$. As implied by the arguments of $S_{l}, \sigma_{l}$, and $\sigma_{\mathrm{s} l}$, we consider only monoenergetic transport; 1.e., we ignore energy as an independent variable. We also restrict our considerations to isotropic scattering. These two restrictions are not essential and are made only for simplicity of exposition. To obtain a formally exact set of statıstical equations, we simply write down, for the particular (but arbitrary) physical realization of the statistics under consideration, a statement of particle balance for each of the two materials in $V$. We subsequently perform an ensemble average of these equations. If we take the volume $V$ to be time independent and fixed in space, our balance considerations constitute an Eulerian derivation.

We define $f(\mathbf{r}, \boldsymbol{\Omega}, t)$ as the distribution function for particles such that $f \mathrm{~d} \mathbf{r} \mathrm{d} \boldsymbol{\Omega}$ is the number of particles in $\mathrm{d} \mathbf{r} \mathbf{\Omega}$ at time $t$. We also define, in the usual way, $\psi(\mathbf{r}, \boldsymbol{\Omega}, t)=v f(\mathbf{r}, \mathbf{\Omega}, t)$, where $v$ is the particle speed. The balance equation for material $i$ in $V$ simply states that the time rate of change of the number of particles, in $i$ and $V$, is the gain rate of particles minus the loss rate of particles. Our considerations constitute a completely standard Eulerian derivation of the transport equation, with due account taken of the existence of two materials in $V$. The fact that two materials are present leads to an additional term in the balance equation for material $i$ involving flow across the interface surfaces $\Gamma$. This term gives the formal coupling between the two materials. 
To facilitate the derivation, we introduce the characterıstic function $\chi_{t}(\mathbf{r}, t)$, defined as

$$
\chi_{1}(\mathbf{r}, t)= \begin{cases}1, & \text { if position } \mathbf{r} \text { is in material } t \text { at time } t \\ 0, & \text { otherwise }\end{cases}
$$

Performing a balance for particles in material $i$ in the volume $V$ gives four standard gain/loss terms, just as in the derivation of the transport equation for a nonstochastic medium. These are:

External source rate

$$
=\int_{V} \mathrm{~d} \mathbf{r} \chi_{1}(\mathbf{r}, t) S_{t}(\mathbf{r}, \boldsymbol{\Omega}, t) \mathrm{d} \boldsymbol{\Omega},
$$

Collision loss rate

$$
=\int_{V} \mathrm{~d} \mathbf{r} \chi_{1}(\mathbf{r}, t) \sigma_{l}(\mathbf{r}, t) \psi(\mathbf{r}, \mathbf{\Omega}, t) \mathrm{d} \mathbf{\Omega},
$$

Scattering gain rate

$$
=(4 \pi)^{-1} \int_{V} \mathrm{~d} \mathbf{r} \chi_{1}(\mathbf{r}, t) \sigma_{\mathrm{s} l}(\mathbf{r}, t) \mathrm{d} \boldsymbol{\Omega} \int_{4 \pi} \mathrm{d} \boldsymbol{\Omega}^{\prime} \psi\left(\mathbf{r}, \boldsymbol{\Omega}^{\prime}, t\right),
$$

Leakage loss rate across the bounding surface $B$

$$
=\int_{B} \mathrm{~d} \mathbf{s} \chi_{1}(\mathbf{r}, t)(\mathbf{n} \cdot \mathbf{\Omega}) \psi(\mathbf{r}, \boldsymbol{\Omega}, t) \mathrm{d} \mathbf{\Omega} .
$$

The vector $\mathbf{n}$ in Eq. (28) is a unit normal outward pointing vector at a local surface point on $B$. Aside from the occurrence of the factor $\chi_{l}(\mathbf{r}, t)$ and the index $\imath$ on $S_{l}, \sigma_{i}$ and $\sigma_{\mathrm{s} i}$ in Eqs. (25)-(28), these are the usual four gain/loss terms which enter into the Eulerian derivation of the classic nonstochastic transport equation. This factor $\chi_{1}(\mathbf{r}, t)$ and the indices extant account for the fact that our balance considerations within the volume $V$ are only for material $t$. In the present context, however, one additional term must be present in the particle balance. This term accounts for the loss and gain of particles in material $i$ due to flow across the internal interface surfaces $\Gamma$. This is given by

Leakage loss rate across the internal surfaces $\Gamma$

$$
=\int_{\Gamma} \mathrm{ds}\left(\mathbf{n}_{i} \cdot \mathbf{\Omega}\right) \psi(\mathbf{r}, \mathbf{\Omega}, t) \mathrm{d} \mathbf{\Omega},
$$

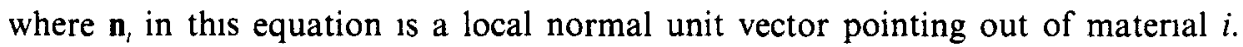

The balance equation for material $i$ in volume $V$ follows by equating the time derivative of the number of particles in material $i$ in volume $V$ and solid angle increment $\mathrm{d} \boldsymbol{\Omega}$ to the sum, with the appropriate sign to account for gains and losses, of Eqs. (25)-(29). Cancelling the differential $\mathbf{d} \boldsymbol{\Omega}$ common to each term, we find

$$
\begin{aligned}
& \frac{1}{v} \frac{\partial}{\partial t} \int_{V} \mathrm{~d} \mathbf{r} \chi_{l} \psi(\boldsymbol{\Omega})=\int_{V} \mathrm{~d} \mathbf{r} \chi_{l} S_{l}(\mathbf{\Omega})-\int_{V} \mathrm{~d} \mathbf{r} \chi_{l} \sigma, \psi(\mathbf{\Omega}) \\
& \quad+(4 \pi)^{-1} \int_{V} \mathrm{~d} \mathbf{r} \chi_{l} \sigma_{s i} \int_{4 \pi} \mathrm{d} \boldsymbol{\Omega}^{\prime} \psi\left(\boldsymbol{\Omega}^{\prime}\right)-\int_{B} \mathrm{~d} \mathbf{s} \chi_{l}(\mathbf{n} \cdot \boldsymbol{\Omega}) \psi(\boldsymbol{\Omega})-\int_{\Gamma} \mathrm{ds}\left(\mathbf{n}_{t} \cdot \mathbf{\Omega}\right) \psi(\boldsymbol{\Omega})
\end{aligned}
$$

In writing Eq. (30), we have dropped the $\mathbf{r}$ and $t$ dependences of all quantities for notational simplicity. This equation can also be obtained by multiplying Eq. (1) by $\chi_{1}(\mathbf{r}, t)$, integrating over the volume $V$, and applying Gauss's theorem.

Equation (30) is an exact balance equation for material $i$ for one particular physical realization of the statistics. The next step is to ensemble average this result over all statistical realizations. To this end, we define

$$
\begin{aligned}
p_{1}(\mathbf{r}, t) & =\left\langle\chi_{l}(\mathbf{r}, t)\right\rangle, \\
\psi_{t}(\mathbf{r}, \mathbf{\Omega}, t) & =\left\langle\chi_{1}(\mathbf{r}, t) \psi(\mathbf{r}, \mathbf{\Omega}, t)\right\rangle\left\langle\left\langle\chi_{t}(\mathbf{r}, t)\right\rangle,\right.
\end{aligned}
$$


where the $\langle\cdot\rangle$ notation means ensemble average. It is clear that $p_{i}(\mathbf{r}, t)$ is simply the probability of finding material $i$ at position $\mathbf{r}$ and time $t$, and $\psi_{1}(\mathbf{r}, \mathbf{\Omega}, t)$ is the ensemble averaged value of the intensity given that position $\mathbf{r}$ is in material $i$ at time $t$. In ensemble averaging Eq. (30), the averaging operator passes inside the volume integrals as well as the integral over the bounding surface $B$, since $V$ and $B$ are common to all physical realizations of the statistics. However, this operator does not pass inside the surface integral over $\Gamma$, since $\Gamma$ depends upon the statistical realization. Taking this into account, an ensemble averaging Eq. (30) gives

$$
\begin{aligned}
& \frac{1}{v} \frac{\partial}{\partial t} \int_{V} \mathrm{~d} \mathbf{r} p_{i} \psi_{i}(\boldsymbol{\Omega})+\int_{v} \mathrm{~d} \mathbf{s}(\mathbf{n} \cdot \boldsymbol{\Omega}) p_{i} \psi_{i}(\boldsymbol{\Omega})+\int_{V} \mathrm{~d} \mathbf{r} \sigma_{i} p_{i} \psi_{i} \\
& =(4 \pi)^{-1} \int_{V} \mathrm{~d} \mathbf{r} \sigma_{\mathrm{s} l} p_{\imath} \int_{4 \pi} \mathrm{d} \boldsymbol{\Omega}^{\prime} \psi\left(\boldsymbol{\Omega}^{\prime}\right)+\int_{V} \mathrm{~d} \mathbf{r} p_{l} S_{l}(\boldsymbol{\Omega})-\left\langle\int_{\Gamma} \mathrm{ds}\left(\mathbf{n}_{i} \cdot \boldsymbol{\Omega}\right) \psi(\boldsymbol{\Omega})\right\rangle .
\end{aligned}
$$

We apply the divergence theorem to convert the integral over the closed surface $B$ to a volume integral, and subsequently take the limit of the entire equation as the volume $V$ approaches zero. We then arrive at our final result given by

$$
\begin{aligned}
& v^{-1} \partial\left[p_{i} \psi_{i}(\mathbf{\Omega})\right] / \partial t+\boldsymbol{\Omega} \cdot \nabla\left[p_{i} \psi_{i}(\boldsymbol{\Omega})\right]+\sigma_{i} p_{i} \psi_{i}(\boldsymbol{\Omega}) \\
& =\left[\sigma_{\mathrm{s} l} /(4 \pi)\right] p_{i} \int_{4 \pi} \mathrm{d} \boldsymbol{\Omega}^{\prime} \psi_{l}\left(\boldsymbol{\Omega}^{\prime}\right)+p_{i} S_{l}(\boldsymbol{\Omega})+\theta_{i}(\mathbf{\Omega}),
\end{aligned}
$$

where we have defined

$$
\theta_{1}(\mathbf{r}, \boldsymbol{\Omega}, t)=-\lim _{\boldsymbol{V} \rightarrow 0}\left[V^{-1}\left\langle\psi(\boldsymbol{\Omega}) \int_{\Gamma} \mathrm{d} \mathbf{s}\left(\mathbf{n}_{1} \cdot \boldsymbol{\Omega}\right)\right\rangle\right]
$$

We note that $\theta_{0}+\theta_{1}=0$ since $\mathbf{n}_{0}=-\mathbf{n}_{1}$. Equations (34) and (35), which hold for each material $i=0,1$, constitute an exact, albeit formal, description of transport in a binary statistical medium for arbitrary statistics. The statistical information is embodied in the probabilities $p_{1}$ and in the coupling terms $\theta_{1}$, which must be evaluated (approximated) to turn this description into a useful computational algorithm. We consider one simple approximation for $\theta_{1}$ in this paper.

It suffices to consider $\theta_{0}$, since $\theta_{1}$ follows from $\theta_{1}=-\theta_{0}$. We consider the integral in Eq. (35) as the sum of two integrals, one for $\mathbf{n}_{0} \cdot \mathbf{\Omega}>0$ and one for $\mathbf{n}_{0} \cdot \mathbf{\Omega}<0$. We then have

$$
\theta_{0}=V^{-1}\left[-\left\langle\psi(\boldsymbol{\Omega}) \int_{\Gamma_{0}} \mathrm{ds}\left(\mathbf{n}_{0} \cdot \boldsymbol{\Omega}\right)\right\rangle+\left\langle\psi(\boldsymbol{\Omega}) \int_{\Gamma_{1}} \mathrm{ds}\left(\mathbf{n}_{1} \cdot \boldsymbol{\Omega}\right)\right\rangle\right],
$$

where we have used $\mathbf{n}_{1}=-\mathbf{n}_{0}$, and the limit $V \rightarrow 0$ is implied in Eq. (36). The notation $\Gamma_{1}$ means the subset of the surfaces composing $\Gamma$ such that $\mathbf{n}_{f} \cdot \mathbf{\Omega}>0$. The two terms in Eq. (36) are of the same form, and it suffices to consider only one of them. Accordingly, we consider the term $T_{0}$, defined as

$$
T_{0}=V^{-1}\left\langle\psi(\boldsymbol{\Omega}) \int_{\Gamma_{0}} \mathrm{ds}\left(\mathbf{n}_{0} \cdot \boldsymbol{\Omega}\right)\right\rangle,
$$

where again the limit $V \rightarrow 0$ is implied. We rewrite Eq. (37) as

$$
T_{0}=\frac{\left\langle\psi(\boldsymbol{\Omega}) \int_{\Gamma_{0}} \mathrm{ds}\left(\mathbf{n}_{0} \cdot \mathbf{\Omega}\right)\right\rangle}{\left\langle\int_{\Gamma_{0}} \mathrm{ds}\left(\mathbf{n}_{0} \cdot \boldsymbol{\Omega}\right)\right\rangle}\left[V^{-1}\left\langle\int_{\Gamma_{0}} \mathrm{ds}\left(\mathbf{n}_{0} \cdot \boldsymbol{\Omega}\right)\right\rangle\right] .
$$

The second bracketed term is simply a geometric quantity dependent upon the statistics, and we define $\Lambda_{0}(\mathbf{r}, \mathbf{\Omega}, t)$ in terms of this quantity as

$$
p_{0} / \Lambda_{0}=V^{-1}\left\langle\int_{\Gamma_{0}} \mathrm{ds}\left(\mathbf{n}_{0} \cdot \mathbf{\Omega}\right)\right\rangle
$$

where $p_{0}(\mathbf{r}, t)$ is the probability of finding material 0 at position $\mathbf{r}$ and time $t$. Equation (38) then becomes

$$
T_{0}=p_{0} \bar{\psi}_{0} / \Lambda_{0}
$$


where we have defined

$$
\bar{\psi}_{0}(\boldsymbol{\Omega})=\left\langle\psi(\boldsymbol{\Omega}) \int_{I_{0}} \mathrm{~d} \mathbf{s}\left(\mathbf{n}_{0} \cdot \boldsymbol{\Omega}\right)\right\rangle /\left\langle\int_{\Gamma_{0}} \mathrm{ds}\left(\mathbf{n}_{0} \cdot \mathbf{\Omega}\right)\right\rangle .
$$

At this point we have made no approximations, Eq. (40) is exact.

To proceed, we note that $\bar{\psi}_{0}$ is an average, in the ensemble average sense, of values of the intensity at interface points, i.e., on $\Gamma_{0}$. We approximate this average by using an analog of upwind differencing encountered in numerical analysis of hyperbolic equations. Specifically, we replace $\bar{\psi}_{0}$ in Eq. (40) with $\psi_{0}$, the ensemble-averaged intensity within material $0 . \psi_{0}$ here is the same $\psi_{0}$ contained in Eq. (34). With this approximation, Eq. (40) becomes

$$
T_{0}=p_{0} \psi_{0} / \Lambda_{0},
$$

and recalling the origin of $T_{0}$ [see Eq. (37)] and the definition of the coupling term [see Eq. (36)], we have

$$
\theta_{0}(\mathbf{r}, \mathbf{\Omega}, t)=p_{1}(\mathbf{r}, t) \psi_{1}(\mathbf{r}, \boldsymbol{\Omega}, t) / \Lambda_{1}(\mathbf{r}, \boldsymbol{\Omega}, t)-p_{0}(\mathbf{r}, t) \psi_{0}(\mathbf{r}, \mathbf{\Omega}, t) / \Lambda_{0}(\mathbf{r}, \boldsymbol{\Omega}, t),
$$

where $A_{1}$ is defined by Eq. (39) with the 0 subscripts replaced with 1 . The coupling term $\theta_{1}(\mathbf{r}, \mathbf{\Omega}, t)$ follows in this approximation from $\theta_{1}=-\theta_{0}$. Using this form of the coupling term in Eq. (34), we see we have reproduced the model given by Eq. (3). This model was first derived using the master equation ${ }^{4.5}$ and a second derivation has recently been given by Sahni. ${ }^{10,11}$ In these two derivations, considerations were restricted to Markov statistics, but the present derivation shows that this model, while approximate, applies to arbitrary statistics. The details of the statistics are embodied in the evaluation of the expression for $\Lambda_{1}$ given by [see Eq. (39)]

$$
p_{t} / \Lambda_{t}=\lim _{V \rightarrow 0}\left[V^{-1}\left\langle\int_{\Gamma_{t}} \mathrm{~d} \mathbf{s}\left(\mathbf{n}_{t} \cdot \mathbf{\Omega}\right)\right\rangle\right] .
$$

This model is known to be exact for time-1ndependent, purely absorbing $\left(\sigma_{\mathrm{st}}=0\right)$ mixtures obeying Markov statistics, and within the context of the present derivation it is clear why this is so. The approximation of replacing the interface ensemble-averaged intensity with the volumetric ensemble-averaged intensity, i.e., setting $\bar{\psi}_{l}=\psi_{l}$, is in this case not an approximation; it is an exact replacement. This observation follows from the fact that for purely absorbing, time-independent transport the solution at any spatial point depends only upon the optical depths from the point in question to the system boundary and the source points. For Markov statistics, the ensembleaveraged optical depth between one spatial point and another is the same if one of the points is an interface, or if this point is chosen at random in one of the materials. Further, in the Markov case, $\Lambda_{t}$ is simply the fundamental Markov transition probability quantity. That is, $\mathrm{d} x / \Lambda_{t}$ is the probability of transition from material $i$ to the other material in a distance $\mathrm{d} x$. These ttems are discussed in detail elsewhere. ${ }^{13}$

The final item we wish to show in this section is that for homogeneous statistics, by which we mean that any point along a particular ray in the medium obeys the same statistics, the parameter $\Lambda$, defined by Eq. (44) can be identified as the mean (average) chord length in material $i$. It suffices to show this for $\Lambda_{0}$, since the arguments for $\Lambda_{1}$ are identical. We consider the evaluation of the quantity $Q_{0}(\mathbf{r}, \mathbf{\Omega}, t)$, defined as

$$
Q_{0}(\mathbf{\Omega})=\lim _{V \rightarrow 0}\left[V^{-1}\left\langle\int_{\Gamma_{0}} \mathrm{ds}\left(\mathbf{n}_{0} \cdot \mathbf{\Omega}\right)\right\rangle\right]
$$

Referring to Fig. 1, we take the direction $\boldsymbol{\Omega}$ to be the $x$ axis, and consider a differential volume of thickness $\mathrm{d} x$ and cross-sectional area $\mathrm{d} A$. Evaluating the ensemble averaged integral in Eq. (45) is equivalent to placing this volume at random on the $x$ axis, computing the integral, and then averaging over all possible random placements. We first note that the randomly placed volume has a probability $p_{0}$ of being in material 0 . Given that the volume is in material 0 , the probability of the volume containing an interface with $\mathbf{n}_{0} \cdot \boldsymbol{\Omega}>0$ is just $\mathrm{d} x / \lambda_{0}$, where $\lambda_{0}$ is the mean (average) chord length in material 0 . Finally, we use the fact that simple geometric considerations give 


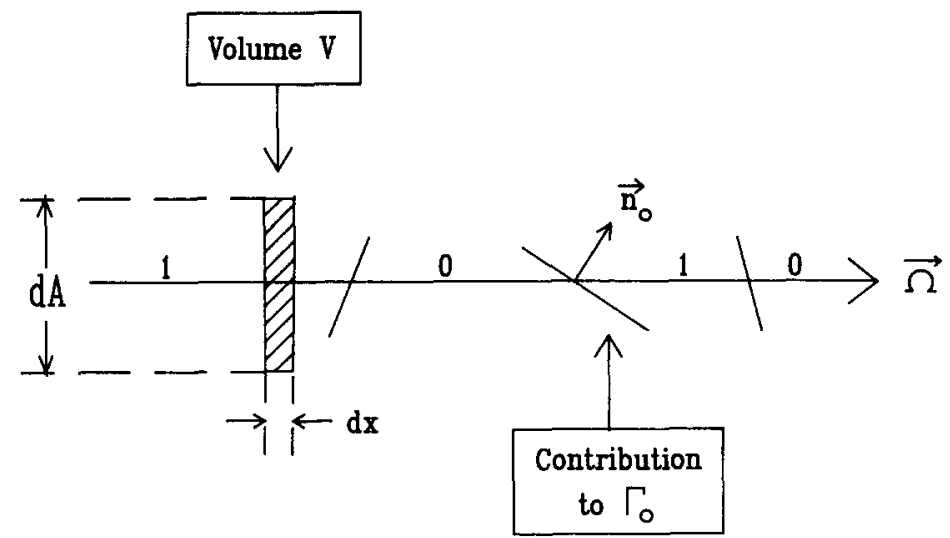

Fig 1 A differential volume placed on a line consisting of alternating material segments 0 and 1

$\mathrm{d} A=\mathrm{ds}\left(\mathbf{n}_{0} \cdot \mathbf{\Omega}\right)$. Putting all of these considerations together, we have

$$
\lim _{V \rightarrow 0}\left[\left\langle\int_{\Gamma_{0}} \mathrm{~d} \mathbf{s}\left(\mathbf{n}_{0} \cdot \mathbf{\Omega}\right)\right\rangle\right]=p_{0} \mathrm{~d} x \mathrm{~d} A / \lambda_{0} .
$$

Since the volume is given by $\mathrm{d} x \mathrm{~d} A$, we then find, using this volume and Eq. (46) in Eq. (45),

$$
Q_{0}=p_{0} / \lambda_{0}
$$

Recalling the definition of $Q_{0}$ [see Eq. (45)], we see that Eq. (47) is identical to Eq. (44) with $t=0$ if one identifies $\Lambda_{0}$ in Eq. (44) with $\lambda_{0}$, the mean chord length in material 0.

In this case of homogeneous statistics, a simple physical interpretation of our approximation to the coupling terms can be given. For homogeneous statistics, we have $p_{i}=\lambda_{1} /\left(\lambda_{0}+\lambda_{1}\right)$, a result written earlier [see Eq. (9)] for homogeneous Markov statistics, but true for any homogeneous statistics. Using this expression for $p_{1}$ in Eq. (43) with $\Lambda_{l}=\lambda_{1}$ and using the fact that $\theta_{1}=-\theta_{0}$, we find that $\theta_{1}$ can be written very simply in terms of the mean chord lengths $\lambda_{r}$ as

$$
\theta_{1}=\left(\psi_{J}-\psi_{1}\right) /\left(\lambda_{0}+\lambda_{1}\right), \quad J \neq i
$$

We also recall that $\theta_{1}$ is the ensemble-averaged rate at which particles flow through $\Gamma$ from material $j$ to material $i$ [see Eqs. (29) and (35)]. With this in mind, we can interpret Eq. (48) as a particular (discretized) form of a Fick's law of diffusion. This law states that the rate at which particles diffuse across an interface is proportional to their concentration gradient at the interface or, more generally, this rate is proportional to the change in concentration across the interface. Fick's law has been applied in many areas of physics, and our approximation to the coupling terms can be physically interpreted as a form of this law.

In future work, we hope to be able to approximate the coupling term $\theta_{t}$ in Eq. (34) more accurately, to produce a better model describing particle transport in a binary statistical medium.

Acknowledgements - The work of the first author (MLA) was performed under the auspices of the United States Department of Energy at the Lawrence Livermore National Laboratory under Contract No. W-7405-ENG-48 The work of the second author (EWL) was partially supported by the Lawrence Livermore National Laboratory as well as through a National Science Foundation grant (EET-8721680). The work of the third author (GCP) was partially supported by the Lawrence Livermore National Laboratory as well as through grants from the National Science Foundation (DMS-8811863) and the Los Alamos National Laboratory (UC-88-4-H-37)

\section{REFERENCES}

1. C. D. Levermore, G. C. Pomraning, D. L. Sanzo, and J. Wong, J. Math. Phys. 27, 2526 (1986).

2. D. Vanderhaegen, JQSRT 36, 557 (1986).

3. D. Vanderhaegen, JQSRT 39, 333 (1988).

4 C. D Levermore, G. C. Pomraning, and J. Wong, J. Math. Phys. 29, 995 (1988).

5. G. C. Pomraning, C. D. Levermore, and J. Wong, Lecture Notes in Pure Applied Mathematics, Vol. 115 , pp. 1-35, P. Nelson et al eds., Marcel Dekker, New York, NY (1989).

6. D. Vanderhaegen and C. Deutsch, J. Statist. Phys. 54, 331 (1989). 
7 G. C. Pomraning, JQSRT 40, 479 (1988).

8 G. C. Pomraning, Transp. Theory Statist. Phys 17, 595 (1988).

9. G C. Pomraning, JQSRT 41, 103 (1989).

10 D. C Sahni, submitted to Ann. Nucl. Energy (1989)

11 D. C Sahni, submitted to J. Math. Phys. (1989).

12. R Sanchez, submitted to J. Math. Phys. (1989).

13. G C Pomraning, JQSRT 42, 279 (1989).

14 J. J. Duderstadt and W R. Martin, Transport Theory, pp. 422-438, Wiley-Interscience, New York, NY (1979) 